\title{
The Potential of Compact Libs System with Multi-Pulse Nd:YAG Laser for Bacteria Identification
}

\author{
A. Ponce Flores ${ }^{1}$, A. K. Frias Sanchez², A. Villarreal'2, F. G. Rendón Sauz², \\ L. Ponce Cabrera'2 ${ }^{2}$ T. Flores Reyes ${ }^{2}$ \\ ${ }^{1}$ Facultad de Ciencias, Universidad Nacional Autónoma de México, México D.F., México \\ ${ }^{2}$ IPN CICATA ALTAMIRA, Carretera Tampico-Puerto Industrial Altamira, Altamira, México \\ Email: aleponce92@ciencias.unam.mx
}

Received 5 January 2015; accepted 19 March 2015; published 26 March 2015

Copyright $@ 2015$ by authors and Scientific Research Publishing Inc.

This work is licensed under the Creative Commons Attribution International License (CC BY). http://creativecommons.org/licenses/by/4.0/

(c) (1) Open Access

\section{Abstract}

This work shows the potential of a very compact, portable Laser Induced-Breakdown Spectroscopy (LIBS) system that includes low cost and compact Nd:YAG laser, intended for use in fieldwork identification of bacteria. This device allows for the generation of $2-4$ pulses per laser shot, with a maximum energy of $120 \mathrm{~mJ}$ per pulse. Each pulse has a duration of $60 \mathrm{~ns}$, and the total duration of the train of pulses is $180 \mu \mathrm{s}$. The device dimensions including the power supply are $50 \times 20 \times 140$ cm, which indicates that can be utilized as a portable LIBS system in field conditions. The spectra captured on two different bacteria shows the capability of identification.

\section{Keywords}

Laser, LIBS, Bacteria, Spectroscopy

\section{Introduction}

Identification of microbiological objects such as bacteria, fungi and pollens has become a critical task for medical, biological and environmental surveillance. Several methods have been proposed for the identification of bacterial strain, based on molecular techniques [1]. Unfortunately, as well know these techniques present difficulties as use of consumables, and also expensive and complex instrumentation [2]. It is also important to remark the importance of sample preparation due the time consumption and the cost. Them, is understandably the increasing need for fast and precise techniques for microbiological samples identification [3].

Between the analytical techniques for microanalysis, Laser Induced-Breakdown Spectroscopy (LIBS) has 
been considered one of the most promising for field applications. This is an atomic emission spectroscopy technique for material characterization that is based on a laser beam that generates plasma upon irradiating a sample. Unlike other atomic emission spectroscopy methods, the source of excitation is a laser pulse, providing various advantages over other techniques. Among these advantages, the system can detect almost any element, does not require prior sample preparation, and enables fast detection; in addition, because of its portability, it is possible to perform an in-situ or nearby analysis for inaccessible samples [4]. Thanks to all these advantages, this technique has gained many applications during the last years [5].

However, this technique has several limitations, including the price and technical complexities of lasers as sources of excitation and the non-optimal detection limits compared with other techniques [6] [7]. Therefore, the detection limits have been improved mainly with increases in the intensity of the plasma emission [8]. Previous works have followed the approach based on the double pulse [9]. Two consecutive laser pulses are incident under different configurations. An extension of this method is the multi-pulse regimen [10], which has the advantage of using passive Q-switches. Q-switches are less expensive, more compact and have a longer lifespan than the cavity switching systems used to generate shorter pulses in double-pulse set-ups. These works have proved that using more than one laser pulse to generate the plasma improves the signal intensity of the LIBS spectrum, which, in turn, improves the detection limit.

LIBS permits rapid analysis without sample preparation, which opens the possibility of fieldwork usage for applications in archeology or forensics. The effectiveness of the technique thus directly depends on the robustness and portability of the equipment. In fact, there are relatively portable LIBS systems. In [11], the development of a portable system is described operating under a multi-pulse regimen. However, the reported increase in the portability and robustness of the instruments is limited by the architecture of the laser cavity. The resonator alignment and relatively large dimensions are key limiting factors.

In this work, we present the design and configuration of a very compact and economic multi-pulse laser that is completely immune to the alignment problem. Its reduced dimensions make its manual utilization possible and allow for its usage in fieldwork. The device uses as excitation source a multi-pulse Q-switched Nd:YAG laser. The emission regimen of this laser causes an enhance of intensity for several spectral lines reinforcing the differences between the spectra of different samples.

Finally, the obtained spectra in reference samples of bacteria demonstrate that the detection quality is adequate and the bacteria recognition is possible by using this system. Rapid identification of bacteria using a portable and inexpensive technique, would have great importance as it could effectively solve the existing demand for express determination in this field.

\section{Experimental Set-Up}

A LIBS set-up is composed of two main elements, a spectrometer and a laser. The most utilized laser is the Nd:YAG type in the Q-switch regime because the high power in the pulse makes it possible to vaporize almost any material. In this work, a very compact Nd:YAG laser is used as the excitation source. The laser allows pulse trains with high peak power to be achieved.

Cr:YAG saturable absorbers have been widely utilized for the switching gain in diode pumped Nd:YAG lasers. Nevertheless, their usage in lamp-pumped lasers has been more limited. In previous works, this type of saturable absorber was used in lamp-pumped lasers with high energy for applications such as thin film deposition [12].

In the equipment developed in this work, the optical cavity contains an active medium in the form of a ND: YAG bar with a 5-mm diameter and 50-mm total length. At one end of the crystal, there is the saturable absorber that constitutes the Q-switch. The Q-switch is composed of a Cd:YAG crystal of 3-mm thickness and initial transmittance of $14 \%$ for a wavelength of $1064 \mathrm{~nm}$. The bar surface is polished while maintaining the parallelism between the sides where the resonator mirrors are placed. One of the mirrors is directly placed over the saturable absorber and has a high reflectance of $99.6 \%$. The second mirror corresponds to the output coupler and has a reflectance of $50 \%$ being placed directly over the active medium. Both mirrors make it possible to obtain the maximum compactness, maintain the alignment and dispense of expensive and unstable long-term adjustable supports, thus reducing design and set-up costs. The pumping of the active medium is realized through a xenon pulse lamp. Its light concentrates over the bar thanks to a monolithic quartz reflector that is coated with a silver layer.

The power supply was developed in a very compact manner $(4 \times 6 \mathrm{~cm})$. Essentially, it is a switched source that loads a capacitor of $500 \mu \mathrm{F}$. E is controlled by a microcontroller that allows the shot energy to be selected. 
Both the optical cavity and power supply are placed inside the ABS plastic case. Figure 2 presents a photograph of the laser. The external dimensions are $20 \times 70 \times 150 \mathrm{~mm}$, and the weight is $304 \mathrm{~g}$. The laser takes the energy from a battery cell, which makes the emission of 50 shots possible without requiring connection to the electricity grid. This feature is a very important aspect for fieldwork analysis. Figure 1 presents a diagram of the experimental LIBS set-up. The laser emits shots with one or several pulses at a wavelength of $1064 \mathrm{~nm}$. The laser beam is focused on the sample through a lens with a focal distance of $10 \mathrm{~cm}$. The temporal structure of the shot is monitored using a RIGOL DS-1102E digital oscilloscope. Upon impinging on the sample surface, the laser causes the formation of the plasma emission, which is collected and introduced into an optical fiber with a diameter of $200 \mu \mathrm{m}$. The fiber conveys the radiation to a Digikrom DK480 1/2 m spectrometer within a detection range of $20 \mathrm{~nm}$ and spectral resolution of $0.03 \mathrm{~nm}$ with an ICCD detector. The delay between the laser shot and spectrometer reading is controlled by a delay generator. This parameter has been set to $1 \mu$ s for all the experiments.

To verify the potential of this LIBS system for microbiological samples identification, laser performance as an excitation source in a LIBS system, we utilized two samples.

One culture of Staphylococcus aureus, and another of Escherichia coli, both bacteria where supplied by from the Instituto de Diagnóstico y Referencia Epidemiológicos of Mexico. For the analysis by LIBS, the samples were placed on a microscope slide.

The identification of the spectral lines was performed using the database published by the National Institute of Standards and Technology (NIST) as a reference.

\section{Results and Discussion}

\subsection{Laser Characterization}

As previously mentioned, the laser generates a pulse train in each shot and for each energy discharge of the lamp. These pulse trains may contain several pulses per shot, with the number of pulses being proportional to the excitation energy. The excitation energy has been limited to ensure only 4 pulses per shot, which facilitates both the characterization of the emission and the study of the plasma excitation. Figure 2 shows the temporal structure of the laser shot for the 2 and 4 pulse cases. The energy per shot according to the number of pulses is 8,17 , 24 and $32 \mathrm{~mJ}$ for shots that contain 1, 2, 3, and 4 pulses, respectively.

As previously mentioned, the laser generates a pulse train in each shot and for each energy discharge of the lamp. These pulse trains may contain several pulses per shot, with the number of pulses being proportional to the excitation energy. The excitation energy has been limited to ensure only 4 pulses per shot for the maximum energy level. The spectra showed in Figure 3, were captured using a 4 pulse laser shot. As can be seen in Figure 2, the inter-pulse separation between the first and second pulse, and also between the second and third pulse, is about 10 microseconds. As was reported previously [12]-[14], for this inter-pulse separation a plasma reexcitation can take place, reinforcing the intensity of selected lines and them, enhancing the signal to noise relationship.

\subsection{LIBS Experiments}

The experiments were performed using the set-up described in Figure 1. The targets were placed such that the in

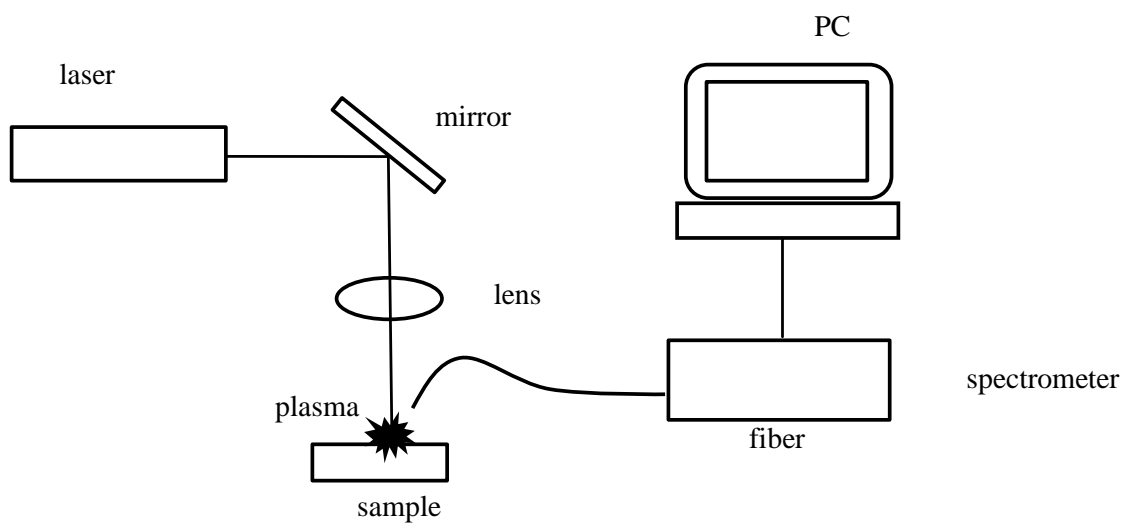

Figure 1. Diagram of the experimental set-up. 


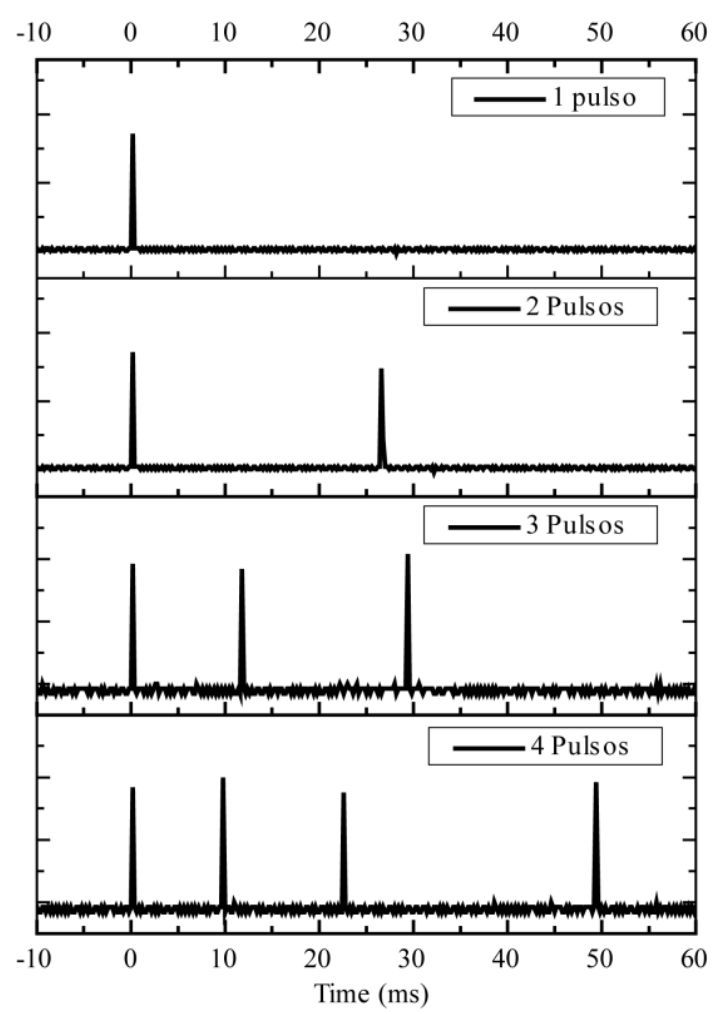

Figure 2. Inter-pulse separation per shot.

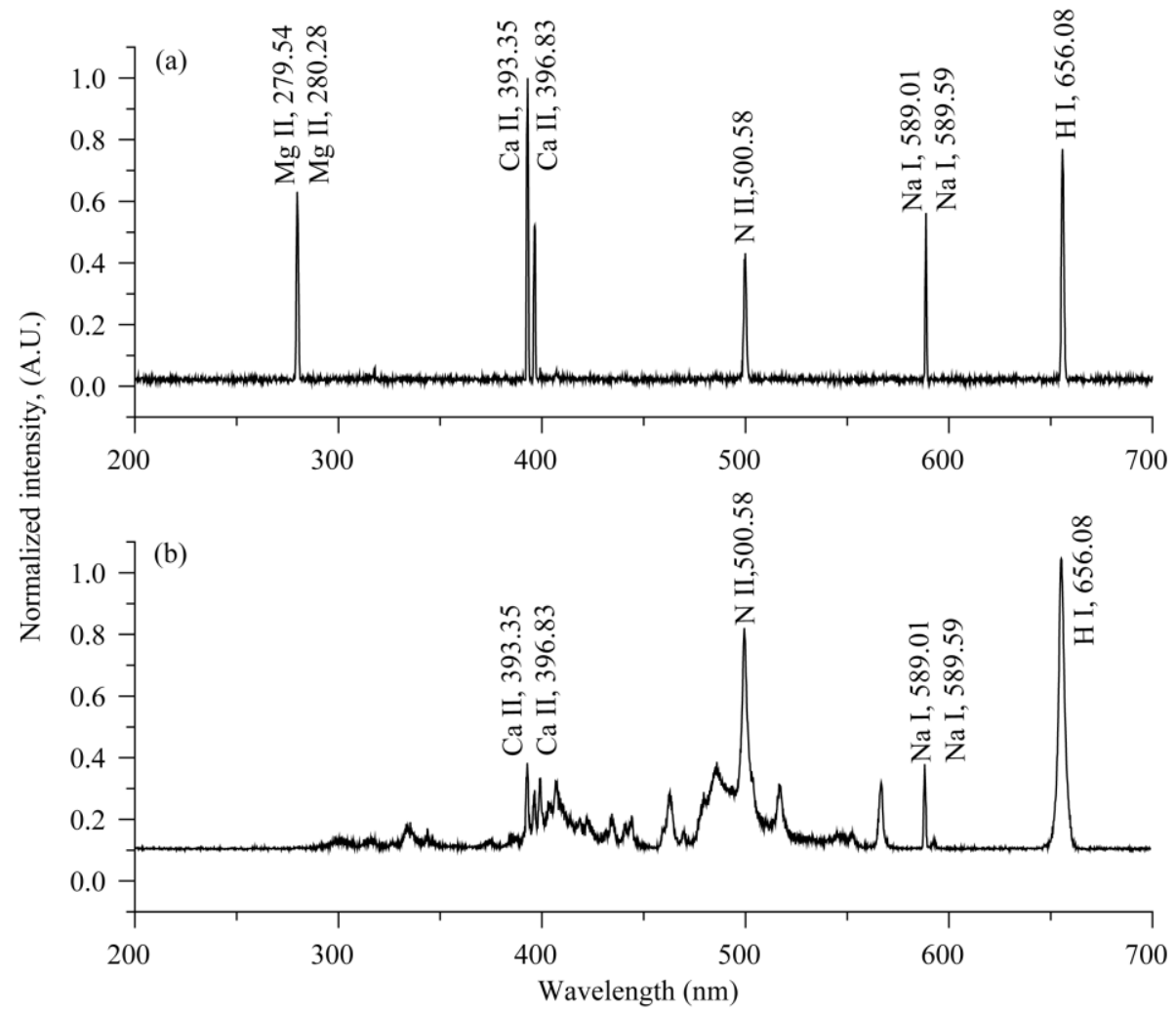

Figure 3. Spectrum of two species of bacteria obtained with a pulse shot. (a) Escherichia coli; (b) Staphylococcus aureus. 
cidence of the laser pulse occurred perpendicularly to the sample surface. The number of pulses per shot was controlled by the excitation energy increase of the pumping lamp. The number of pulses is proportional to the total energy because the energy per pulse is approximately $8 \mathrm{~mJ}$ regardless of the number of pulses per shot. The laser shots were performed over a non-irradiated surface, avoiding the presence of the crater generated by a previous shot.

Figure 3 presents time-integrated spectra obtained in the LIBS set-up using multi-pulse laser shots with four pulses per shot. Both spectra are recorded with a detection delay of $10 \mu$ s. In this overview presentation of the spectra, one can already observe clear differences between both spectra. In first place, the most intense pikes correspond to ionized species [13]. The possibility of exciting ionized lines by multi-pulse regime has been reported previously [14]. This is very important to identify the species of bacteria, since it is possible to use ionized lines, typically more complicated to reveal, for example CII and MgII.

In Figure 3, we can see clearly that the emissions from both MgII and CaII are significantly larger in Escherichia than in Staphylococcus aureus. As very well know, the first of these species is a Gram-negative while the second one is a Gram-positive. The outer membrane of Gram-negative bacteria contents proteins that are maintained together via divalent cations, such as MgII and CaII. For this reason, it's expected to find more intense magnesium and calcium emission from Escherichia coli, which is confirmed by experiments.

\section{Conclusions}

We have developed a Nd:YAG laser with Q-switches and a saturable absorber for application in portable LIBS for in-situ analysis. The design is very compact, battery powered without risk of misalignment and capable of emitting in a multi-pulse regime.

The device allows for the emission of several pulses in a single laser shot. In addition, the number of pulses per shot as well as the inter-pulse separation can be programmed. This feature allows improvement of the intensity of the emission signal as well as gathering of information on the ablation processes and the temporal evolution of the generated plasma under these conditions.

The spectra captured on biological micro-samples, shows the potential of this technique for express determination of bacteria strains. It is remarkable that the information from the spectra obtained by this method allows, in principle, identify bacteria without a mathematical processing. Rapid identification of bacteria using a portable and inexpensive technique could effectively solve the existing demand for express determination in this field.

\section{Acknowledgements}

The authors would like to thank the UNELAB from Universidad del Noreste for supply the samples. This work was financed by CONACYT-Fondo Mixto Tamaulipas.

\section{References}

[1] Kostic, T., Butaye, P. and Schrenzel, J. (2009) Detection of Highly Dangerous Pathogens: Microarray Methods for BSL 3 and BSL 4 Agents. John Wiley \& Sons, New York. http://dx.doi.org/10.1002/9783527626687

[2] Snydman, D.R. (1992) Book Review. New England Journal of Medicine, 327, 1326-1326. http://dx.doi.org/10.1056/NEJM199210293271825

[3] Wang, D., Coscoy, L., Zylberberg, M., Avila, P.C., Boushey, H.A., Ganem, D., et al. (2002) Microarray-Based Detection and Genotyping of Viral Pathogens. Proceedings of the National Academy of Sciences, 99, 15687-15692.

[4] Rusak, D.A., Castle, B.C., Smith, B.W. and Winefordner, J.D. (1997) Fundamentals and Applications of Laser-Induced Breakdown Spectroscopy. Critical Reviews in Analytical Chemistry, 27, 257-290.

[5] Cremers, D.A. and Radziemski, L.J. (2013) Handbook of Laser-Induced Breakdown Spectroscopy, Elemental Analysis. 2nd Edition, Wiley Online Library. http://dx.doi.org/10.1002/9781118567371

[6] Hahn, D.W. and Omenetto, N. (2012) Laser-Induced Breakdown Spectroscopy (LIBS), Part II: Review of Instrumental and Methodological Approaches to Material Analysis and Applications to Different Fields. Applied Spectroscopy, 66, 347-419. http://dx.doi.org/10.1366/11-06574

[7] Pasquini, C., Cortez, J., Silva, L.M.C. and Gonzaga, F.B. (2007) Laser Induced Breakdown Spectroscopy. Journal of the Brazilian Chemical Society, 18, 463-512.

[8] Radziemski, L.J. (1994) Review of Selected Analytical Applications of Laser Plasmas and Laser Ablation, 1987-1994. Microchemical Journal, 50, 218-234. 
[9] Gautier, C., Fichet, P., Menut, D., Lacour, J.L., L’Hermite, D. and Dubessy, J. (2005) Quantification of the Intensity Enhancements for the Double-Pulse Laser-Induced Breakdown Spectroscopy in the Orthogonal Beam Geometry. Spectrochimica Acta - Part B Atomic Spectroscopy, 60, 265-276. http://dx.doi.org/10.1016/j.sab.2005.01.006

[10] Le Drogoff, B., Margot, J., Vidal, F., Laville, S., Chaker, M., Sabsabi, M., et al. (2004) Influence of the Laser Pulse Duration on Laser-Produced Plasma Properties. Plasma Sources Science and Technology, 13, 223-230. http://dx.doi.org/10.1088/0963-0252/13/2/005

[11] Elsayed, K., Imam, H., Harfoosh, A., Hassebo, Y., Elbaz, Y., Aziz, M., et al. (2012) Design and Construction of Q-Switched Nd:YAG Laser System for LIBS Measurements. Optics \& Laser Technology, 44, 130-135.

[12] Peñaloza-Mendoza, Y. and Ponce-Cabrera, L. (2014) Comparison on Morphological and Optical Properties of TiO 2 Thin Films Grown by Single-Pulse and Multi-Pulse Laser Ablation. Journal of Surface Engineered Materials and Advanced Technology, 5, 17.

[13] Morris, J.A. and Pollock, C.R. (1990) Passive Q Switching of a Diode-Pumped Nd:YAG Laser with a Saturable Absorber. Optics Letters, 15, 440-442. http://dx.doi.org/10.1364/OL.15.000440

[14] Galbács, G., Jedlinszki, N., Herrera, K., Omenetto, N., Smith, B.W. and Winefordner, J.D. (2010) A Study of Ablation, Spatial, and Temporal Characteristics of Laser-Induced Plasmas Generated by Multiple Collinear Pulses. Applied Spectroscopy, 64, 161-172. 